\title{
Smith-magenis syndrome. A unique neonatal presentation among the Arab population
}

\begin{abstract}
Smith-Magenis Syndrome (SMS) is a rare multisystem genetic disorder caused by a heterozygous deletion of or a heterozygous pathogenic variant in RAI1 on chromosome 17p11.2. characterized by the variable intellectual deficit, sleep disturbance, brachycephaly, midface hypoplasia, prognathism, hoarse voice, speech delay with or without hearing loss, psychomotor and growth retardation, cutaneous features, and behaviour problems. Our reported case is a term newborn diagnosed antenatally in the feto-maternal unit to have large multi-cystic dysplastic left kidney and unilateral cerebralventriculomegaly. The genetic disorder was suspected due to symmetric IUGR and dysmorphic features. Chromosomal micro-array confirmed the diagnosis of Smith-Magenis Syndrome.MRI brain confirmed Dandy-Walker spectrum malformation. Ultrasound abdomen showed left multi-cystic dysplastic kidney.
\end{abstract}

Smith-Magenis Syndrome is usually diagnosed in childhood and little is known about its presentation in neonates especially in the Arab population. Our patient presented with Intracranial haemorrhage, seizures, thrombocytopenia, feeding difficulty, hypoglycemia and acute kidney injury; which all resolve before discharge.

Keywords: brachycephaly, midface hypoplasia, prognathism, ventriculomegaly
Volume 10 Issue 6 - 2020

\author{
Alaa Masry, Mahmoud Alkhdr,Husam Salama, \\ Nuha Nimeri \\ Hamad Medical Corporation ,Women's Wellness and research \\ Centre, Qatar
}

Correspondence: Nuha Nimeri, Hamad Medical Corporation, Women's Wellness and research Centre NICU, Qatar, Email nuha@usa.net

Received: December 14, 2020 | Published: December 29 2020

\section{Case presentation}

We report a rare case of a newborn male, who was born at term. The antenatal scan was normal for twin A whose examination was normal after delivery. DNA banking sent for her with no further investigations done depending on FMU findings and clinical examination post delivery. Twin B was found to have multicystic dysplastic kidney disease, cerebral ventriculomegaly with normal cardiac structure. This newborn needed intensive resuscitation including intubation due to increased fio 2 requirement and desaturation. He was shifted to NICU for further management.

The initial examination showed small for age newborn, his growth parameters were all below 3rd centile donating intrauterine growth restriction. Grossly dysmorphic with wrinkled skin, flexed posture, abnormal head shape, high forehead, depressed nasal bridge anteverted nostrils, low set ears, micrognathia, proptosis, thick eyelashes and eyelids, partial syndactyly of 2nd and 3rd toes bilaterally with flattened feet and hands. The abdomen was bulging with palpable hard left upper quadrant mass, about $4 \mathrm{~cm}$, liver edge also felt below costal margin. The newborn was connected to mechanical ventilation for one day, extubated to CPAP, weaned to nasal cannula for another 3 days then he was off respiratory support till discharge. First $x$ ray showed homogeneous soft tissue opacity occupying the left abdominal cavity pushing the bowel loops to the right side most likely the left enlarged kidney(Figure 1). Ultrasound scan revealed average size of both hepatic lobes with smooth surfaces, coarse hepatic parenchymal texture with diffuse periportal thickening, and extensive thick GB sludge. The Left Kidney was hugely enlarged Multicystic dysplastic with multiple sizable cortical cysts. It measures $6.7 \times 3.8 \mathrm{~cm}$; right kidney was normal (Figure 2).

The newborn developed acute kidney injury manifested as oliguria with impaired renal function, conservative management with input output charting was done. Over few days renal function normalized, and it was attributed to perinatal asphyxia.

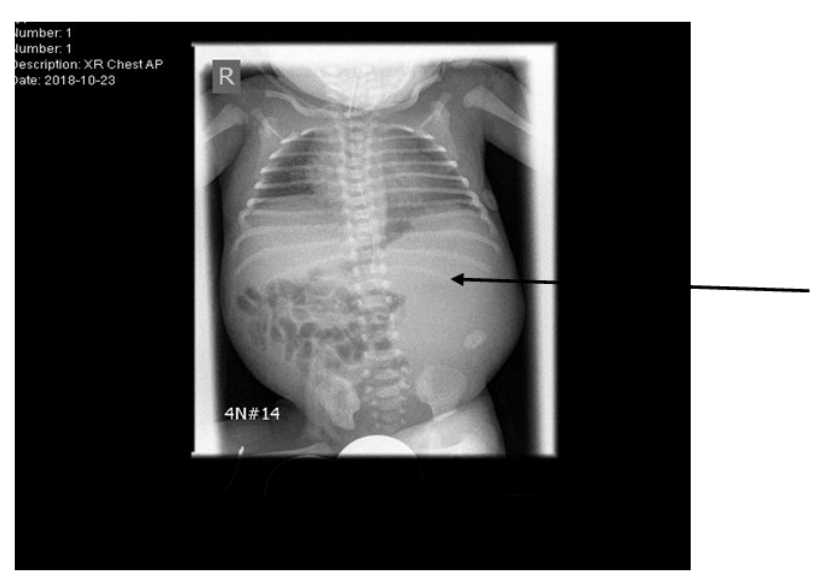

Figure I Chest and abdomen $X$ ray showing homogeneous soft tissue opacity is occupying the left abdominal cavity pushing the bowel loops to the right side.

Brain ultrasound showed Bilateral grade I IVH with small hematomas at germinal matrix regions and small cerebral hematoma noted in the right occipital lobe measures about $11 \times 14 \mathrm{~mm}$ with prominent cisterna magna(figure 3). MRI scan at age of 5 days revealed Posterior fossa retro cerebellar cyst suggestive of DandyWalker spectrum malformation, Intracranial hemorrhage with right occipital and left occipitoparietal hematomas, bilateral cerebellar microhemorrhages and intraventricular hemorrhage and Mild to moderate supratentorial hydrocephalus(figure 4). The patient developed severe Thrombocytopenia started on the first day. Platelet count was 23,000 needed frequent PLTs transfusions, improved within 4 days to 123,000 . The patient developed seizures at two weeks with lip smacking controlled well by Phenobarbitone. Neurosurgery and neurology team managed intracranial bleed conservatively. Last brain ultrasound: no detectable new fresh blood clots seen, Lateral ventricular 
dilatation. $\mathrm{V} / \mathrm{H}$ ratio $=32: 84 \mathrm{~mm}$, Residual degenerated blood clots in the occipital horn of left lateral ventricle measures $12 \times 10 \mathrm{~mm}$, redemonstrations of Dandy-Walker spectrum malformation changes.

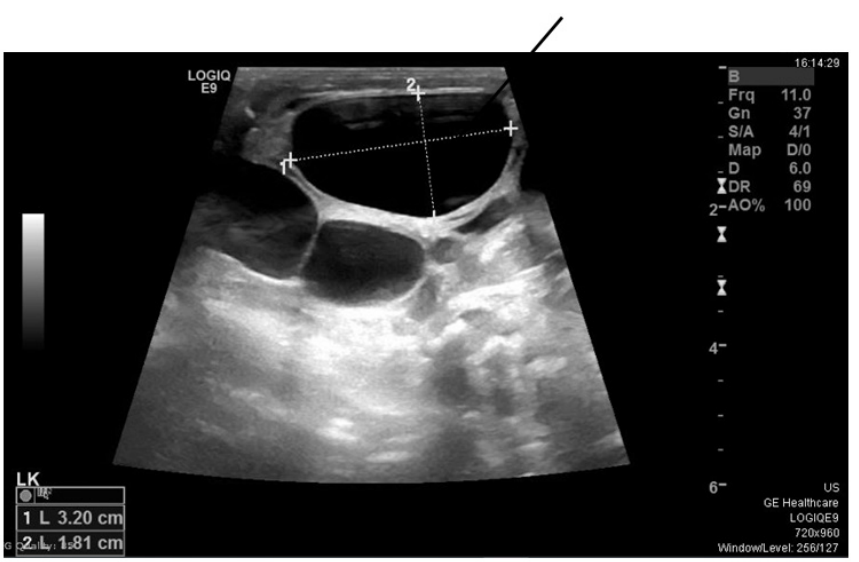

Figure 2 Ultrasound abdomen showing Hugely enlarged Multicystic dysplastic left kidney with multiple sizable cortical cysts.

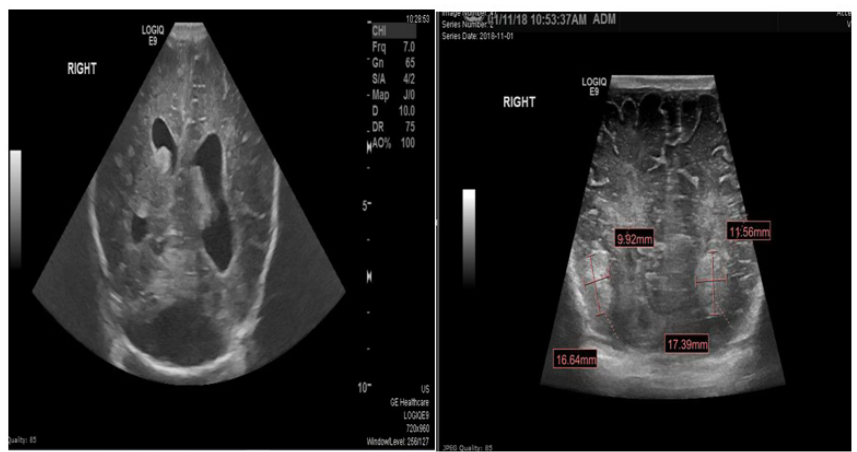

Figure 3 Ultrasound Brain showing Resolving bilateral grade II IVH with small degenerated blood clots in the lateral ventricles Posterior fossa retro cerebellar cyst suggestive of Dandy-Walker spectrum malformation. Bilateral occipital cerebral hematomas starting degeneration measure $17 \times 10 \mathrm{~mm}$ on the right side and measures $17 \times 12 \mathrm{~mm}$ on the left side.

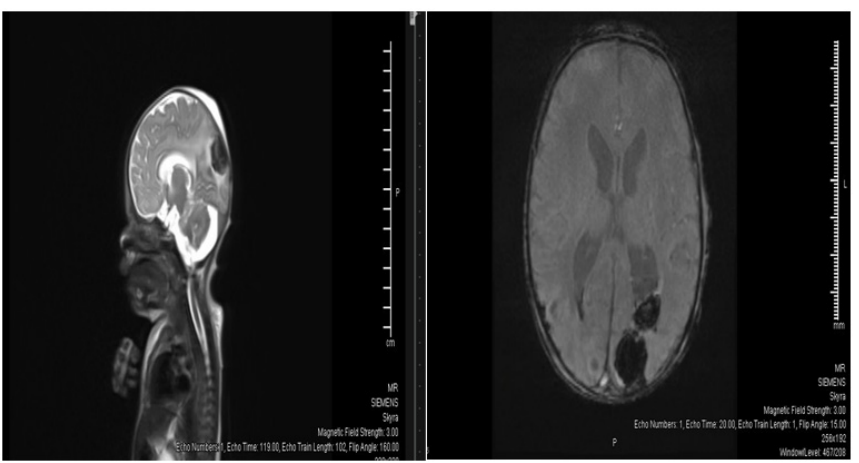

Figure $4 \mathrm{MRI}$ brain Posterior fossa retrocerebellar cyst suggestive of Dandy-Walker spectrum malformation. Mild to moderate supratentorial hydrocephalus.

The patient had persistent Hypoglycemia needed prolonged high dextrose concentration. Dextrose $20 \%$ together with adding polycose to 24 calories milk were used. The condition gradually improved. Critical samples were sent, including growth hormone, $\mathrm{TSH}$, cortisone, c-peptide and ketone bodies and were all normal. The reasons were not clear, yet it didn't recur after that.

Microarray analysis showed an interstitial deletion of $\sim 3.63 \mathrm{Mb}$ within cytogenetic band $17 \mathrm{p} 11.2$. This is associated with Smith-
Magenis syndrome (SMS) (OMIM 182290). The deleted segment includes RAI1, AKAP10, ALDH3A2, ATPAF2, FLCN, MYO15A.

The family was counselled by the genetic team. They explained the clinical manifestations of the syndrome and recurrence risk in subsequent pregnancies.

TORCH screen was normal. Blood gases were normal after the initial period of renal impairment. Skeletal survey together with hip US normal. Eyes examination by ophthalmologist came normal.

The patient needed occupational therapist and physical therapist intervention for sucking training and within 4 weeks the baby was able to have full feeding by oral sucking. The infant was discharged home in stable condition on full oral feedings. There was planned follow up in different subspecialties clinics. The patient died after 9 month at home, the reason for death was not known most likely sudden infant death syndrome. The parents were seen in the family bereavement clinic and all needed support was given. The other twin discharged home in a good condition.

\section{Discussion}

Smith-Magenis Syndrome (SMS) is a microdeletion syndrome characterized by an abnormality in the short (p) arm of chromosome 17 and is sometimes called the $17 \mathrm{p}$ - syndrome. ${ }^{1}$

The estimated frequency of SMS is $1 / 25,000$, but it is quite likely that the syndrome is underdiagnosed because of its relatively recent description and requirement for high-resolution cytogenetics and/or FISH to detect the deletion. ${ }^{2}$

It was first reported in the medical literature in 1982 by Ann Smith. ${ }^{3}$ In 1986, Smith and Dr. R. Ellen Magenis identified nine patients with the disorder and further delineating the features of the syndrome. Since that time few additional cases have been identified.

Among the Arab Community the first Case of SMS in Nazareth was reported by Nijim Y et al., 2016. ${ }^{4}$ This's the first case to be reported in state of Qatar from non-Arabian descent.

Smith- Magenis syndrome usually present later in childhood. Few is known about its presentation during neonatal period.

A complex disorder, SMS, is a contiguous-gene deletion syndrome that comprises characteristic clinical features, developmental delay, peripheral neuropathy, abnormal sleep function, and phenotypical characteristics affecting the behavioral, neurological, and physical features in an individual. ${ }^{5,6}$

The features of SMS are likely to range in severity from person to person (variable expressivity). SMS is an unpredictable and randomly occurring condition that has no identifiable risk factors; it occurs in roughly 1 in 15,000 to 25,000 births and was first described by Ann C. M. Smith and her colleagues in $1982.5,6$

Two unrelated patients with facial clefts and congenital heart defects (CHD) were first reported in 1982. Various clinical phenotypes have been described in patients with interstitial deletion of $17 \mathrm{p} 11.2$ (Smith et al., 1986), and subsequently defined as a deletion syndrome in 1986 by Stratton et al.

The diagnosis of SMS is based on clinical findings and confirmed by genetic testing. Microarray analysis can confirm either a deletion of chromosome $17 \mathrm{p} 11.2$, which encompasses multiple genes including the retinoic acid-induced 1 gene (RAI1) or mutations in the RAI1 gene itself. With an estimated prevalence of 1:15,000 to 25,000, SMS is not genetically inherited. ${ }^{7,8}$ 
The facial appearance is characterized by a broad square-shaped face, brachycephaly, prominent forehead, synophrys, mildly up slanted palpebral fissures, deep-set eyes, broad nasal bridge, midfacial retrusion (formerly known as midfacial hypoplasia), short, full-tipped nose with reduced nasal height, micrognathia. ${ }^{9}$ This facial phenotype almost matching with (high forehead, brachycephaly, mid face hypoplasia, depressed nasal bridge up slanting palpebral fissure, ant everted nostrils, low set ears, micrognathia).

A variety of cardiac defects, including septal defects, valvular abnormalities, and tetralogy of Fallot have been described in patients with Smith-Magenis syndrome.

Heart defects are identified in fewer than $50 \%$ of affected individuals with SMS who have a deletion of $17 \mathrm{p} 11.2$ but have not been reported in those who have a heterozygous pathogenic variant in RAI1. Cardiac anomalies may include mild tricuspid or mitral valve stenosis or regurgitation, ventricular septal defects, supravalvular aortic or pulmonic stenosis, atrial septal defects. ${ }^{10}$

After delivery, some of the reported cases didn't reach acceptable saturation and needed respiratory support .[10] Exactly as we found in our case but our patient didn't have major cardiac anomalies only PFO with PDA and was discharged with scheduled follow up with cardiologist.

The feature of micro and brachycephaly, is one of the characteristics of SMS. The only known neuropathologic study on a patient with Smith-Magenis syndrome in whom the entire $17 \mathrm{p} 11.2$ band was deleted, demonstrated microcephaly and foreshortened frontal lobes with neuronal depletion; a small choroid plexus hemangioma was also observed in the lateral ventricle. ${ }^{11}$

Nonspecific central nervous system structural abnormalities documented by neuroimaging may be observed in over half of affected individuals, with an increased frequency of ventriculomegaly reported. ${ }^{11}$ In our case the FMU scan revealed ventriculomegaly and features of Dandy-Walker spectrum malformation. Intracranial haemorrhage with Rt.occipital and left occipitoparietal haematomas, bilateral cerebellar microhemorrhages and intraventricular haemorrhage were also found in our patient.

However, there is no other specific neuroimaging finding associated with Smith-Magenis syndrome. Computed tomographic scans performed in 25 patients with Smith-Magenis syndrome demonstrated ventriculomegaly in 9, enlargement of the cisterna magna in 2 , and partial absence of the cerebellar vermis in one patient. ${ }^{12}$

Similar findings were observed among a group of 10 children who had undergone previous magnetic resonance imaging: 5 had ventriculomegaly; 2 had enlarged posterior fossa; and 3 had normal scans. ${ }^{13}$

Feeding difficulties occurs in SMS in general as well as our reported case; leading to failure to thrive are common, including marked oral motor dysfunction with poor suck and swallow and textural aversion.

Genitourinary anomalies are found in between $15 \%$ and $35 \%$ of affected individuals who have a deletion of $17 \mathrm{p} 11.2$ but have not been reported in those who have a heterozygous pathogenic variant in RAI1. ${ }^{14}$ The presence of left multi-cystic dysplastic kidney which a unique association was not reported before in literature.

This case was not from Arabian descent and travelled after discharge to his home country, and unfortunately passed at age of 9 months with no identifiable cause for us.
We couldn't comment on the behavioral findings that happen later in childhood, since the baby died early. In comparison to our case present and diagnosed during the neonatal period.

Our aim behind reporting this case is to describe clinical manifestations, signs, complications and management of a rare syndrome especially it presented for the first time in state of Qatar.

The aim of this paper is to share the experience we gained in handling this baby, the atypical clinical presentation which leads to the diagnosis. To highlight the management options and to review similar cases from literatures.

He had unique presentation with new association not reported before namely the multi-cystic dysplastic kidney, the dandy walker malformation and the multiple intracranial bleeds together with the unexplained persistent hypoglycemia.

\section{Conflict of interest}

The author declares no conflict of interest.

\section{Acknowledgments}

None.

\section{Funding}

None.

\section{References}

1. Weimin Bi,Jiong Yan, Paweł Stankiewicz, Sung-Sup Park, et al. Genes in a refined Smith-Magenis syndrome critical deletion interval on chromosome 17p11.2 and the syntenic region of the mouse. Genome res. 2002;12(5):713-728.

2. Juyal RC, Figuera LE, Hauge X, et al. Molecular analyses of $17 \mathrm{p} 11.2$ deletions in 62 Smith-Magenis syndrome patients. American journal of human genetics. U.S. National Library of Medicine. Am J Hum Genet. 1996;58(5):998-1007.

3. Greenberg F, Guzzetta V, Montes de Oca-Luna R, et al. Molecular analysis of the Smith-Magenis syndrome: a possible contiguousgene syndrome associated with $\operatorname{del}(17)(\mathrm{p} 11.2)$. Am J Hum Genet. 1991;49(6):1207-1218.

4. Nijim Y, Adawi A, Bisharat B, et al. First Case Report of SmithMagenis Syndrome (SMS) Among the Arab Community in Nazareth: View and Overview. Medicine. Wolters Kluwer Health; Medicine. 2016;95(3):e2362.

5. Bass MN, Speak BL. A behavioural approach to the assessment and treatment of severeself-injury in a woman with Smith-Magenis syndrome: a single case study. Behav Cogn Psychother. 2005;33:361368.

6. Poisson A, Nicolas A, Cochat P, et al. Behavioral disturbance and treatment strategies in Smith-Magenis syndrome. Orphanet Journal of Rare Diseases. 2015;10:111.

7. Gropman A, Duncan W, Smith A. Neurologic and Developmental Features of the Smith-Magenis Syndrome (del 17p11.2). Pediatric Neurology. 2006;34(5):337-350.

8. Sloneem J, Oliver C, Udwin O, et al. Prevalence, phenomenology, aetiology and predictors of challenging behaviour in Smith-Magenis syndrome. J Intellect Disabil Res. 2011;55(2):138-151.

9. Adam MP, Ardinger HH, Pagon RA, et al., editors. Seattle (WA): University of Washington, Seattle. 1993-2019. 
10. Myers S, Challman T. Congenital heart defects associated with SmithMagenis syndrome: Two cases of total anomalous pulmonary venous return. Am J Med Genet. 2004;131(1):99-100.

11. Smith A, McGavran L, Robinson J, et al. Interstitial deletion of (17) (p11.2p11.2) in nine patients. Am J Med Genet. 1986;24(3):393-414.

12. Wolters P, Gropman A, Martin S, et al. Neurodevelopment of Children Under 3 Years of Age With Smith-Magenis Syndrome. Pediatric Neurology. 2009;41(4):250-258.
13. Martin SC, Wolters PL, Smith AC. Adaptive and maladaptive behavior in children with Smith-Magenis Syndrome. J Autism Dev Disord. 2006;36(4):541-552.

14. Myers S, Challman T, Bock G. End-stage renal failure in SmithMagenis syndrome. American Journal of Medical Genetics Part A. 2007;143A(16):1922-1924. 